\title{
9. COMMISSION DES INSTRUMENTS ASTRONOMIQUES
}

\author{
Président: M. J. A. Carroll, Admiralty, Whitehall, London, S.W. I, England.
}

Membres: MM. Anderson, R. Baillaud, J. G. Baker, Burch, Chrétien, Couder, Danjon, Dimitroff, Dufay, Dunham, J. W. Evans, Gaviola, Hargreaves, Henyey, Lallemand, Linfoot, Link, Lyot, McKellar, McMath, Maksutov, Minnaert, Óhman, Perfect, Plaskett, Redman, Rösch, Ross, Rosseland, Schilt, Sternberk, Whitford, Yü.

\section{New Observatories and Telescopes}

The 200-inch telescope at Mt Palomar in California is complete and in process of adjustment prior to commencing a programme of observations. The large mirror, after successful figuring, has been transported to the observatory without mishap and it is expected that some months at least will be spent on adjustments to its supports and in testing its performance and that of the associated gear.

The Royal Observatory, Greenwich, is to move to Herstmonceux near the south coast of England. The conditions at Greenwich have deteriorated steadily over many years and the new site offers as good conditions climatically as can be found in Great Britain and is well removed from industrial glare or fog as there are no towns near the new site, which consists of a fine old moated brick castle in some 200 acres of ground.

A start has been made on the conversion of the castle and on new buildings for instruments. For some years observations will be carried on at both Greenwich and Herstmonceux.

Two new meridian instruments are under construction for the Royal Observatory, a horizontal transit instrument and an improved photographic zenith tube. In the horizontal transit instrument the telescopic system is stationary at all times. The system consists of two identical objectives having their optical axes coincident. These objectives face east and west respectively (that is to say their common axis is horizontal and normal to the meridian) and the distance between them is equal to the sum of their focal lengths.

Observations of a star are made by placing a two-mirror optical square alternately in front of each objective, before and after transit respectively. The fiducial wires in the common focal plane of the objectives are observed first from one side and then from the other.

Apart from the gain in stability due to the fixity of the telescopic system, the essential advantage of the instrument is that the adjustments of the optical square are not critical. Consequently, compared with what is demanded in the pivots of a normal transit instrument, the stringency of mechanical requirements in the only movable part of the instrument is enormously reduced.

This instrument has these further noteworthy features:

(I) The two objectives are mounted kinematically and are provided with micrometer adjustments for focusing and squaring. The mounts themselves are provided with additional adjusting means, consisting of a combination of micrometer screws and spring strips, to enable one objective to be moved vertically and the other horizontally. By these means the line joining the second nodal points of the two objectives can be adjusted so as to be precisely normal to the meridian.

(2) A mechanically driven achromatic optical wedge of variable deviation is mounted in front of the optical square. The direction of the stellar beam entering either objective is thereby maintained axial and errors due to maladjustment are minimized.

(3) The time-scale is produced photographically by means of an autocollimation system applied to mirrors attached to the moving elements of the wedge. It is thus made free from error due to mechanical imperfection in the motion of these elements.

(4) The adjustment and determination of level of the telescopic system is effected by means of nadir observations made successively on mercury reflectors placed beneath the two positions of the optical square. The azimuth is adjusted by successive approximation 
and is afterwards determined in the normal manner. The equivalent of collimation error is eliminated provided the deviation due to the optical square is constant during the observation of a star.

The improved photographic zenith tube differs in the following respects from previous instruments of its type:

(I) The angle of reversal is observed by autocollimation instead of by means of a divided circle. Thus a fixed axis of rotation is unnecessary and accordingly the 'rotary' (the assembly which rotates in azimuth) is supported on a ball-bearing with plane races, greatly reducing friction and facilitating construction. At the same time, undesired rotation or tilting about horizontal axes is kept within the required limits. There being no definite constraint in the horizontal direction, it is possible to define each of the two working orientations by a pair of stops instead of by a single stop as in instruments provided with a fixed axis of rotation.

(2) Provision of adjusting means in the kinematic mounting of the objective enables the nodal plane and the plane of the photographic plate to be brought into precise coincidence.

(3) Automatic reversal is accomplished by means of a system of wires which exert a pure torque on the rotary and therefore no tilting torque on the tube. This system is such that unidirectional rotation of the driving shaft is converted into reciprocating rotation of the driving rotary. At each reversal, moreover, the angular velocity is initially and finally zero, as is the case in the Washington instrument.

(4) The diffraction pattern of the star image is rendered symmetrical by making the plate carriage annular, this carriage encircling the objective. The asymmetrical carriage constraints can therefore be made external to the aperture, while the inevitably obstructing plate-holder and its supports are easily made symmetrical with respect to the aperture. This annular construction, moreover, permits of more effective constraint of the carriage.

(5) Relative motion of the carriage and rotary is made to approximate to pure translation by means of a compensating system of flexed rods which constrain the carriage in the horizontal plane to which the motion is restricted by means of three balls that roll between horizontal plane races. This system secures good repetition of track, which is the essential requirement, and a good approximation to rectilinearity as is desirable.

(6) A method of achieving uniformity of rate in the relative translation of carriage and rotary is under investigation. In this method a differential roller and metallic tapes replace a direct screw drive.

(7) The time-scale is produced photographically by means of a clock-controlled lamp. Erratic mechanical linkage and backlash are thus avoided, as is also the use of an independent chronograph. Duplication of this system not only reduces error in the timescale but provides a check on the repetition of carriage-track and will therefore probably be adopted.

(8) The shutter operates on the incident beam, above the objective, instead of operating on the reflected beam below the plate. It is a symmetrically-opening rotary shutter with provision for timing.

(9) As criterion of adjustment for constancy of scale-value, an optical null method has been introduced for use in conjunction with a suspended silica rod.

A new photographic zenith tube is being constructed at the U.S. Naval Observatory. The lower part will be enclosed in a well containing a pillar supporting the mercury trough, so that the working region at the top of the tube is conveniently accessible from ground level. The cell holding the two components of the 8-inch objective is fitted with stops and a spring to secure positive location of the lenses in a fixed position relative to their cell. Means are provided for rapid checking that the angle of reversal does not differ from $I 80^{\circ}$ by more than five seconds of arc. The operation of the instrument is semiautomatic through a system of controlling relays. The mechanical design and method of manufacture incorporate several novel features.

The government of the U.K. has provided funds for the construction of a new roo-inch reflecting telescope to be called the Isaac Newton Telescope. It will be erected in the Royal Observatory at Herstmonceux and maintained by the observatory for the use of 
British astronomers in general, the observing programme and claims for use of the telescope being under the control of a Board of Management nominated by the Royal Society and the Royal Astronomical Society.

The design of the telescope and its auxiliary gear is at present under discussion by the Board of Management. Very detailed consideration is being given to a form of universal telescope usable at will for wide-field photography, by means of a correcting plate or for axial spectroscopy. A new form of mounting termed an Inverted Fork Equatorial has been proposed and is being closely considered.

The design of this mounting is a collocation of features not new in themselves, and represents an attempt to satisfy three requirements, namely: (x) free access to any part of the sky, (2) great rigidity and stability, and (3) a minimum of two additional reflections (a total of four reflections) in the path of the light to the Coudé focus. The polar axis is a cone with a rim at its wider, upper end running on pressure-oil bearings. Projecting from the upper end of this cone is a short, unsymmetrical hollow column on one side of which is a large annular bearing on which the tube rotates. The counterweight, rotating on a bearing at the other side of the column, is rigidly attached to the upper part of the tube by connections external to the column, rendering unnecessary the usual declinationaxis spindle. Consequently the interior of the column is unobstructed and two diagonals, one in the tube and the other in the column, direct the light down the interior of the polar-axis cone. Alternatively, the column may house a Cassegrain spectrograph supplied with light from the first of the two diagonals.

Among other novel features under consideration is a scheme to correct automatically for the changing deflections of the main telescope tube as its position changes.

A new astrophysical observatory has been erected in Haute-Provence to the south-west of Forcalquier by the Centre National de la Recherche Scientifique. The project was started towards the end of $193^{8}$, under an organizing committee presided over by M. Danjon, Director of the Paris Observatory. The director of the new observatory is M. Dufay, Director of the Observatory of Lyons and M. Fehrenbach of the Strasbourg Observatory is deputy director and resident astronomer in charge. The observatory is at an altitude of 600 metres and enjoys excellent observing conditions and has grounds of some 95 hectares. Three domes, $12 \mathrm{~m} ., 8 \mathrm{~m}$. and $6.5 \mathrm{~m}$. in diameter, house respectively a telescope of $\mathrm{r} \cdot 2 \mathrm{~m}$., another of $8 \mathrm{I} \mathrm{cm}$., and an equatorial mounting. A large laboratory will be equipped during this year.

The $\mathrm{I} \cdot 2 \mathrm{~m}$. telescope has an old mirror from the Paris Observatory refigured by M. Couder. The telescope of $8 \mathrm{I} \mathrm{cm}$. aperture has been used to study the qualities of the stellar images obtainable with well-known excellent results.

The equatorial mounting based on plans by $\mathrm{M}$. Couder comprises a large table of $\mathrm{r} .5 \mathrm{~m}$. side, sufficiently big to carry a telescope of $80 \mathrm{~cm}$. aperture. At present a normal field direct-vision objective prism is mounted.

It is intended to install a telescope of $\mathrm{I} \cdot 93 \mathrm{~m}$. aperture housed in a dome of $24 \mathrm{~m}$. diameter and there is hope that this instrument may be in action in 1952 .

There are two spectroheliographs, one a conventional type and another with one of M. Lyot's filters registering continuously monochromatic solar images.

At the Pic-du-Midi M. Jules Baillaud has erected the $6 \mathrm{I} \mathrm{cm}$. large Coudé objective from the Paris Observatory. The focal length of the objective is $I 8.25 \mathrm{~m}$. but the equatorial tube of the new mounting is kept to only $6 \mathrm{~m}$. by using two plane mirrors. The performance is excellent. An account of the instrument will be published in $L$ 'Astronomie.

The 74-inch mirror for the Radcliffe Observatory, Pretoria, has at last been completed for shipment to South Africa. The mounting and dome have been ready since 1938 .

In Canada in I940 a new observatory was given to the University of Western Ontario by Mrs Frances Amelia Cronyn in memory of her husband the late Hume Cronyn. The telescope is a ro-inch refractor and has a small Schmidt type camera attached.

In Sweden the Uppsala Observatory has received a gift from Dr Nils Tamm which has provided for a station in Kvistaberg, some $40 \mathrm{~km}$. south-west of Uppsala. The main instrument will be a Schmidt telescope of about 40 inches aperture. 
Preparations are being made to extend the equipment of the Stockholm Observatory with a Schmidt telescope of 26 inches aperture and an objective prism of the same size. Several instruments for solar research are under construction including a spectroheliograph and monochromators.

It is intended to add to the existing equipment of the Helwan Observatory a new large reflector of about 94 inches aperture and a new photographic zenith tube of the same type as that for the Royal Observatory, Greenwich.

\section{Developments in Optical Design and Optical Components}

In the fabrication and working of optical components the most generally applicable new technique introduced since 1938 has been the blooming of surfaces by deposition in vacuo of a thin layer of material of suitable refractive index whereby the reflection of light incident on a refracting surface is reduced to a tenth or less of the reflection by the untreated surface. The deposition of reflecting films of aluminium has gained favour and is now widely used for mirrors up to the very largest.

The principal source for the manufacture of very large disks of low expansion glass at the present time appears to be the firm of Pilkington in Great Britain, where disks up to 60 inches diameter can now be cast and annealed, and within a year capacity up to Ioo inches will be available. The St Gobain Works are not at present making large disks but may in the course of a year or so be able to undertake disks up to $2 \mathrm{~m}$. diameter. In Germany at Jena, Zeiss and Schott have resumed manufacture of colour filters and will also be able to undertake manufacture of disks of low expansion glass up to $80 \mathrm{~cm}$. diameter. In the U.S. during the war a number of large crystals of calcium fluoride were grown in sizes up to some $15 \mathrm{~cm}$. Some of these have been deposited with the U.S. Bureau of Standards and might be made available for optical parts for special purposes.

In the field of telescope design, the most striking development has been the widespread adoption of the Schmidt optical system. Corrector plates up to 48 inches in diameter have been constructed, the largest to date being that of the Palomar 48-72-inch Schmidt telescope. The possibility of flattening the field of the Schmidt camera by adding a convex secondary mirror to the system has been discussed in a number of papers by J. G. Baker, H. Slevogt, C. R. Burch and E. H. Linfoot, and telescopes incorporating this modification are under construction for the St Andrews University Observatory, Scotland, and for the Armagh-Harvard-Dunsink station at Bloemfontein. To obtain the sharpest possible images, at least one of the mirrors must be non-spherical, but in some practical cases it is possible to keep both the mirrors spherical without loss of photographic performance.

A. Bouwers, D. Maksutov, A. Colacevich and others showed independently that a meniscus lens, nearly concentric with the spherical mirror surface and of nearly zero power, can effectively replace the Schmidt corrector plate in some designs. This is an advantage in small and medium-sized systems, since the meniscus lens, though rather sharply curved, is much easier to make than a Schmidt plate. Maksutov also designed meniscus-Cassegrain telescopes consisting of a meniscus lens, a concave primary mirror and a convex secondary mirror, and using only spherical surfaces. The practical usefulness of the meniscus design is probably limited at present to apertures not exceeding about I 5 inches by the difficulty of producing large, steeply curved meniscus lenses. The thin aspheric plates of large Schmidt and Schmidt-Cassegrain telescopes can be made from selected sheets of ordinary plate glass, though special glass is also used.

Optical designs giving still higher performance in medium and small sizes have been obtained by combining a concentric meniscus system with an aspheric corrector plate which deals with the residual errors left by the meniscus. Such meniscus-Schmidt cameras have been designed by Bouwers and by Hawkins and Linfoot, with monochromatic errors only about I/I6 of those of the classical Schmidt camera. C. G. Wynne obtained comparable performance without the use of an aspheric surface by means of two near-concentric meniscus lenses, one on each side of the centre of curvature of the spherical mirror; his systems would seem to be especially well suited to spectrograph cameras. 
Other new spectrograph camera designs are the solid Schmidt, which can work down to $f / 0 \cdot 4$ or even lower, and an interesting system of M. Rösch, consisting of a plano-concave lens, its concave surface facing towards, and concentric with, a concave mirror. Constructed with aperture $75 \mathrm{~mm}$., focal length $60 \mathrm{~mm}$., this is said to be usable over a $60^{\circ}$ field. Miniature Schmidt of 2- or 3-inch aperture and of speeds near $f / \mathrm{r}$ are coming into more general use; in particular, one is being constructed by the Ealing Optical Company for the Cambridge University Observatory.

In co-operation with the Aga Company, Stockholm, Prof. B. Lindblad has devised a nebular spectrograph using a Schmidt $f / 0.6$ as a camera and a parabolic mirror as a collimator.

At Meudon M. F. Baldet has had constructed by the Société Générale d'Optique of Paris an objective of $\mathrm{I} 7 \mathrm{~cm}$. focal length, aperture ratio $\mathrm{f} / \mathrm{I}$. The field is some $20^{\circ}$ and stellar images are sharp over about $6^{\circ}$. It will be used for cinematographic study of comets, using $35 \mathrm{~mm}$. film.

An important and interesting development is the normal field objective prism devised by $M$. Charles Fehrenbach. It is a direct vision prism which allows, by returning the rays along the optic axis of the instrument the application of Pickering's method for determining radial velocities, but no field correction is required.

Seidel's and the chromatic aberrations of the Schmidt camera from a new standpoint have been studied theoretically by T. Matukuma (Tohoku Univ.) (to be published in Mem. Astr. Soc. Japan). H. Hirose (Tokyo Astr. Observatory) has directed the construction of Schmidt cameras in Japan. An $\mathrm{f} / \mathrm{I}$ camera of $\mathrm{r} 8 \mathrm{~cm}$. aperture is now under test at the Tokyo Astr. Observatory. H. Hirose and Y. Kobayashi (Kyoto Univ.) studied, independently, optical systems consisting of a spherical lens and a spherical mirror. The theoretical performance of these systems was verified by actual construction.

At Tokyo Astr. Observatory, H. Kubota (Tokyo Univ.) and Y. Yatabe (Fuji Chemical Industry Co.) have collaborated in the reproduction of diffraction gratings with methylacryl-resins. There remain some difficulties yet. (Applied Physics, 16, 68, 1947; Shizen, I947, no. Io-both in Japanese.)

\section{Developments in Mechanical Design of Observing Instruments}

M. Chalonge of the Paris Institute of Astrophysics has devised a mechanical means of broadening stellar spectra which allows the broadening to be varied from $0.3 \mathrm{~mm}$. near 3 Ioo A. to $0.9 \mathrm{~mm}$. near $\mathrm{H} \alpha$.

M. d'Azambuja installed at Meudon in I94I an arrangement allowing spectroheliogrammes in $\mathrm{H} \alpha$ to be taken on $35 \mathrm{~mm}$. film to show by subsequent cinema projection movements in the solar chromosphere, particularly the movements of prominences.

\section{NeW or Greatly Improved Instruments and Technigues}

By far the most outstanding advance in observational astronomy for very many years has come from the application of developments in radio to the study of celestial phenomena. Towards the end of the war it was noticed that on certain short-wave highly directional radio receivers the background noise showed a very strong increase in certain directions and it was found that there was an incoming stream of radiation apparently of galactic origin and also that there were from time to time strong emissions of solar origin. The equipment originally used for these observations was not specially designed for such purposes but was normal service equipment. Since the original discoveries a number of workers have taken up investigation of these phenomena using first modified service equipment and later specially designed equipment of which the principal special features have been directed towards a lower or more stable set background noise or special directional aerial arrays. Some photographs displaying the equipment used and the results obtained by British pioneer workers will be on view during the assembly. 
The other striking advance has been the use of radar for observation of meteor streams whereby it is possible to observe them in much greater detail and over longer times than heretofore and of course observations can be made in the daytime whereby swarms otherwise unobservable can be detected. Determination of velocity and of trajectory is possible now with great accuracy.

It is interesting that the first radar detection of meteors occurred during the use of radar for detecting ' $V$ ' weapons approaching Great Britain during the war.

Attempts are in hand to use radar to measure changes in lunar distance and possibly to effect determination of the absolute value of lunar distance at any time.

To turn to developments on more conventional lines, some of the most striking have been in the field of photoelectric photometry due to the development of successful commercial electron multiplier tubes and the development of sensitive infra-red detectors such as the lead sulphide cell. Various workers have constructed equipment for attachment to telescopes incorporating these devices for astronomical photometric observations or have applied them to laboratory photometers and microphotometers. At the National Observatory, Ondrejov, Czechoslovakia, Dr Link has made use of an anti-aircraft searchlight to make photoelectric measurements of the twilight and night sky, using an electron multiplier cell furnished by M. Lallemand of the Paris Observatory, and placed at the focus of a parabolic mirror of diameter $2 \mathrm{~m}$. and focal length $84 \mathrm{~cm}$. The incident light is interrupted by a rotating sector in order to allow the use of an A.c. amplifier, the amplified current being rectified for measurement. At the Leiden Observatory very successful photometric measurements have been made with a multiplier tube and an extensive programme is being carried out particularly on RR Lyrae in connection with spectroscope research at the Yerkes and McDonald Observatories. The sensitivity is amply sufficient to allow the use of colour filters so that variable stars may be followed in several colours. At Utrecht the photoelectric installation has been tried with incident light interrupted by a rotating sector to allow A.C.: amplification.

Prof. Lyot has developed a number of devices. He has constructed a polarizing filter described by him in I935, which allows observation over a field of about $2^{\circ}$ in a region of the spectrum some $2 \mathrm{~A}$. or $3 \mathrm{~A}$. wide centred about two coronal radiations at $5303 \mathrm{~A}$. and $6374 \mathrm{~A}$. or chromospheric radiations $\mathrm{H} \beta, \mathrm{D}_{3}, \mathrm{~B}_{1}$ and $\mathrm{H} \alpha$. With this he is able to photograph the corona in its own monochromatic radiations with exposures only of a few minutes. By reducing the transmission band to $\mathrm{I} \cdot 5 \mathrm{~A}$. he has been able to take cinema photos of the chromosphere up to the edge of the disk with sufficiently short exposures to get good images with a focal distance equivalent to $20 \mathrm{~m}$. He has also used the polarizing filters to separate radiations and make cinematograph records simultaneously on three different films of the prominences in $\mathrm{H} \alpha$, the corona in $6374 \mathrm{~A}$. and the corona in $5303 \mathrm{~A}$. $\mathrm{He}$ has also devised a direct-current amplifier which can cope with the extremely feeble electromotive force developed by thermocouples. The stability of this instrument is stated to exceed that of galvanometers and its sensitivity not to be limited by the Johnson effect in the circuit to which it is connected. With it can be detected a radiation of $10^{-10} \mathrm{~W}$. It was mounted, in I94I, on the coronagraph at the Pic du Midi for a search for coronal infra-red radiations. No description of this equipment has so far been published because of war-time circumstances, but it is described in documents deposited with the Academy of Sciences in I94I. He has also used multiplier photo-cells and constructed a photoelectric polarimeter which allows the determination in a few seconds of a proportion of polarized light of one I0,oooth part in a luminous flux of one microlumen. He has also constructed a photometer for coronal studies allowing the comparison of the intensity of coronal and solar radiations. A description of this device has not yet been published.

At the Pic du Midi M. Camichel has constructed a micrometer for the measurement of the apparent diameters of small objects. This is achieved by bringing into the field of view the image of the star under study encased between two images of a small circular aperture of known diameter reflected by a glass disk. By a polarizing device colour and brightness of the two images of the aperture can be varied and the diameter modified until they have precisely the same appearance as that of the object under examination. The device has 
been applied to satellites of Jupiter and to the five satellites of Saturn using a $6 \mathrm{I} \mathrm{cm}$. refractor and it has given the diameters of these satellites with a probable error of one or two hundredths of one second.

A new microphotometer has been constructed at Utrecht Observatory, devised in the main by Dr Houtgast. The coupling between the plate and the drum is by means of a lever of variable ratio and the rectilinear movement of the lever is converted into a rotation of the recording drum by simple contact between a flat metal plate and a rotating cylinder, a gentle pressure being sufficient to avoid any slipping. The Philips Factories in Holland are now making Schmidt cameras of low price for television, the correcting plate being made out of gelatine by a special method. The image quality is good, the plates being devised either for an object at infinity or for an object at a distance of $2 \mathrm{~m}$. At Utrecht they are using a small camera of this type in combination with a big prism for observation of the night sky.

The projection micrometer of the Dominion Astrophysical Observatory at Victoria, B.C., described in Journal of the Royal Astronomical Society of Canada for I937, has been built with improved optical and mechanical features and has operated satisfactorily now for a number of years. At the same observatory R. M. Petrie has devised a projection comparator for rapid measurements of plates for determination of radial velocities. The machine is now in its final form, scales have been prepared for various dispersions and various spectral classes of stars. A preliminary description of it was given at a meeting of the American Astronomical Society in 1946 and there is a brief description in Sky and Telescope for 1946. At Victoria there has been constructed a semi-automatic intensitometer designed by C. S. Beals which allows the profiles of spectral lines to be derived rapidly and satisfactorily. A new Littrow form of the Victoria Stellar Spectrograph has been constructed.

For the Stockholm Observatory Dr Y. Öhman has devised a sensitive photoelectric colorimeter applying the flicker effect which appears when light is transmitted through a rotating polarizer and a fixed analyser. The measurements of colour are made by using two intermediate quartz plates, one cut parallel and one perpendicular to the axis. When the polarizer rotates two different spectral regions are alternately transmitted and by turning the analyser a setting may be found for which the alternating current of the phototube disappears. With a suitable rotatory dispersion and sensitivity range this provides a useful colour equivalent.

At the Institute of Theoretical Astrophysics, Blindern, Norway, plans for a new Solar Observatory have been worked out. The Institute's I 2 Unit Differential Analyser has been provisionally completed and is now in use after having been idle through the war years.

At the Observatory at Toulouse, M. Paloque has constructed an astrograph of $150 \mathrm{~mm}$. aperture and $\mathrm{x} \cdot 72 \mathrm{~m}$. focal length. A reduction of plates taken shows residuals corresponding to a probable error two or three times less than those given by meridian observations.

Dr Link reports modifications to a Hale type spectrohelioscope at Ondrejov, including the introduction of a grid system engraved on the metallic plate in the plane of the first slit to allow precision measurements. To observe the profiles of $\mathrm{H} \alpha$ he introduces a photoelectric multiplier cell of $\mathrm{I}_{4}$ stages and fed after amplification to the vertical plates of an oscillograph on the screen of which can be observed directly the profile of the line and its evolution during chromospheric eruptions.

At Bordeaux M. Rösch has constructed an instrument in which stars of different magnitudes are made to stand out at different distances in a stereoscopic comparator. The apparatus allows star counts to be made between assigned magnitudes without the necessity of counting the number of each magnitude. By making two exposures, one through a thin prism and the other through the prism turned through $180^{\circ}$, it is possible to obtain stereoscopic indication of effective wave-length to an accuracy of about $5 \mathrm{~A}$.

At Meudon M. Servajean has constructed a photo-mechanical machine which reproduces selected portions of normal spectroheliograms either on Mercator's projection or in rectangular co-ordinates. 
A new photometer for the measurement of star images by means of an iris micrometer has been constructed for the Rutherfurd Observatory. The beam sent through the plate is equalized to the control beam coming from the same light source by opening or closing the iris; a photoelectric cell follows using A.C. amplification to serve as the indicator of equality of the beams.

\author{
J. A. CARRoll \\ President of the Commission
}

\title{
Report of meeting
}

President: Dr J. A. Carroll.

The Draft Report was adopted with some additions and amendments incorporated.

The Commission, in opening its session, paid respect to the memory of its former president, M. Ch. Fabry, a man outstanding in the field of physical optics and the father of the present very fertile school of younger French workers.

The Commission also recorded their appreciation of the exhibition of instruments and of photographs and diagrams of new astronomical instruments and records obtained with them and congratulated Prof. M. Waldmeier on its successful organization and display.

The President drew the attention of members of the Commission to the possible value of a big improvement in accuracy of line profile determinations. In the last decade or two much effort and ingenuity has gone to facilitating determination of profiles to an accuracy of about $2 \%$ and several devices have been designed to analyse spectra in large numbers with great speed to this order of precision. For some kinds of analyses of line profiles, however, a reduction of the error to $0.2 \%$ or better is needed and it would be of great value to develop means for determining at least a few lines in some stars to a high degree of precision.

At the request of the International Optical Union the attention of astronomers is drawn to the need for co-ordinating requirements for diffraction gratings and to the need felt by manufacturing opticians for selecting the most promising designs from among the very many variations made possible by recent developments of Schmidt cameras and lensmirror systems generally.

For diffraction gratings the Optical Section of the Swedish National Committee for Physics will act as a co-ordinator and centre of information. Inquiries should be addressed to Dr E. Inglestam, Royal Institute of Technology, Stockholm 26, Sweden.

Dr E. Linfoot, Solar Physics Observatory, Cambridge, would be glad to receive views on lens-mirror systems and will attempt co-ordination of designs and requirements.

On the afternoon of Monday, August r6, Dr Hubble spoke informally on the 200-inch reflector. He described the new system of supports, devised in connection with the ribbed structure of the mirror, and the drive, which corrects automatically for refraction and flexure. Observations will not be begun before the tests are completed.

The prime focus, which will be used mainly for direct photography and spectrography without temperature control, will be in use first. The Coude focus is intended for fixed spectrographs of dispersion down to $50 \mathrm{~A} . / \mathrm{mm}$. Of the proposed programmes the cosmological problem will involve the establishment of good photometric sequences of nebular magnitudes, and work on the red-shifts and on the distribution of nebulae. The abundance problem will demand work with the highest dispersion at the Coudé focus.

It is expected that the radius of visibility will be doubled, as compared with the Ioo-inch, this being partly due to improvement in photographic plates. 